\title{
Volumetric Diffuse Optical Tomography for Small Animals Using a CCD-Camera-Based Imaging System
}

\author{
Zi-Jing Lin, ${ }^{1}$ Haijing Niu, ${ }^{2}$ Lin Li, ${ }^{1}$ and Hanli Liu ${ }^{1}$ \\ ${ }^{1}$ Department of Bioengineering, Joint Graduate Program between University of Texas at Arlington and \\ University of Texas Southwestern Medical Center, University of Texas at Arlington, Arlington, TX 76019, USA \\ ${ }^{2}$ State key Laboratory of Cognitive Neuroscience and Learning, Beijing Normal University, Beijing 10875, China
}

Correspondence should be addressed to Hanli Liu, hanli@uta.edu

Received 15 July 2011; Revised 21 October 2011; Accepted 24 October 2011

Academic Editor: Javier A. Jo

Copyright (c) 2012 Zi-Jing Lin et al. This is an open access article distributed under the Creative Commons Attribution License, which permits unrestricted use, distribution, and reproduction in any medium, provided the original work is properly cited.

\begin{abstract}
We report the feasibility of three-dimensional (3D) volumetric diffuse optical tomography for small animal imaging by using a CCD-camera-based imaging system with a newly developed depth compensation algorithm (DCA). Our computer simulations and laboratory phantom studies have demonstrated that the combination of a CCD camera and DCA can significantly improve the accuracy in depth localization and lead to reconstruction of $3 \mathrm{D}$ volumetric images. This approach may present great interests for noninvasive 3D localization of an anomaly hidden in tissue, such as a tumor or a stroke lesion, for preclinical small animal models.
\end{abstract}

\section{Introduction}

Over the last decade, diffuse optical tomography (DOT) has become an emerging medical imaging modality $[1,2]$, which permits near infrared (NIR) light $(650-900 \mathrm{~nm})$ to penetrate deep tissues in several centimeters noninvasively. This technique has been investigated for several clinical applications, particularly for monitoring of functional brain activities [3-5] and detection of breast cancer $[6,7]$. The fundamental principle of DOT is that the detected NIR signals reflect optical properties of the underlying biological tissues $[8,9]$ and that the spatial distribution of such measured optical properties can be used to reconstruct optical tomographic images.

In the meantime, small animal models have been often utilized in preclinical research in order to investigate a variety of human diseases. Commonly used modalities to image small animals include magnetic resonance imaging (MRI) [10], computed tomography (CT) [11], positron emission tomography (PET) [12], and single photon emission computed tomography (SPECT) [13]. As compared to MRI and CT, DOT is able to provide functional sensitivity; as compared to CT, PET, and SPECT, DOT is radiation-free. More recently, DOT has become an imaging methodology in preclinical studies for certain diseases, such as stroke [14] and cancer [15].

To date, fiber-based and charge-coupled device- (CCD-) camera-based DOT techniques are well developed and widely performed in both human and animal studies. A fiberbased DOT system has several advantages, namely, being compatible with different geometry and shape of a measured organ and having low noise because of direct contact of fibers on the tissue surface. However, a limited surface space on small animals is often a constraint to place many fiber optodes and thus restricts the spatial resolution of reconstructed images. A CCD-camera-based DOT system eliminates such a problem since the CCD camera can serve as a detector array with possible thousands of virtual detectors and cover a wide field of view (FOV). Moreover, such a DOT system is simpler and more portable with lower cost, as compared to a fiber-based DOT system.

While DOT is a promising tool to image diseases noninvasively, one major limitation exists due to scattering nature of photons traveling in tissue. Namely, strong light scattering of biological tissue causes the detection sensitivity of DOT to attenuate exponentially with increased depth, resulting in poor depth localization in DOT. A variety of efforts have been made by different research groups to improve the accuracy 
in depth localization. A layer-based sigmoid adjustment (LSA) method was introduced to balance the sensitivity contrast in depth by directly adjusting the forward sensitivity matrix [16]. Spatially variant regularization (SVR) [17] was also introduced to compensate the decrease in measurement sensitivity with increase of depth by modifying the penalty term of regularization along depth. SVR has been utilized in the frequency-domain and CW-based DOT techniques for imaging human breast cancer and brain functions. Recently, we have developed a depth compensation algorithm (DCA) to significantly improve the accuracy of DOT in depth localization, as demonstrated by both laboratory phantom and human brain measurements $[18,19]$, using fiber-based DOT systems.

In this study, we wish to demonstrate the feasibility and improvement of three-dimensional (3D) volumetric DOT for small animal imaging by using CCD-camera-based DOT in combination with DCA. Our results are based on computer simulations and laboratory phantom experiments, confirming that the combination of a CCD camera and DCA is an effective approach for $3 \mathrm{D}$ volumetric images to be utilized in preclinical research.

\section{Materials and Methodology}

2.1. Implementation of a CCD-Camera-Based DOT System. We implemented a CCD-camera-based, NIR spectroscopic imaging system, which consisted of a broadband light source, a multiplexer, a 6-filter wheel, and a high-speed CCD camera (Figure 1(a)) with a pixel matrix of $496 \times 656$. Such a system allowed us to acquire the transmitted/reflected NIR signals from living tissues at specific wavelengths so as to obtain hemodynamic signals within a time scale of a hundred milliseconds. We performed CCD-camera calibration by utilizing laboratory tissue phantoms (Figure 1(b)) in order to (a) calibrate the CCD camera for its nonuniformity and to (b) examine the stability and linearity of the calibrated camera.

\subsection{Correction of the CCD-Camera-Based DOT System.} Numbers of factors affect the results of a CCD image. These factors include (1) small electric signals seen by the camera without any optical input, or dark current, (2) pixel-topixel sensitivity variations, and (3) nonuniform illumination of the CCD chip due to vignetting and dust. Flat field correction is a common technique [20] that can be used to obtain improved quality of CCD images. The flat field technique removes the effects due to dark current, pixelto-pixel sensitivity variations, uneven illumination, and nonuniformity caused by vignetting and dust.

During the process of correction, we collected two types of images besides taking raw object images: (1) dark images without any optical input and (2) reference images with planer illumination. The dark images were needed to correct the electric current that flows through each pixel of the CCD array without any optical input. The planer-illuminated reference images were obtained by uniformly illuminating a highly reflecting reference plate, which has a reflectivity of
$98 \%$ and a dimension of $200 \times 200 \mathrm{~mm}^{2}$. To approximately achieve a planer illumination, we used a white light lamp and placed it 1.5 meters away from the measurement site, with a good amount of light intensity, without saturating the camera.

The procedures used to perform flat field correction in this study are listed below: first, take a dark image by blocking the camera's entrance completely, followed by taking object images with a point-source illumination from one side of the object (see Figure 1(b)). The integration times were kept the same for both situations. Second, take a reference image with a planer illumination followed by the corresponding dark image, also using the same integration time for both. Then, corrected object images can be obtained with the following equation:

$$
I=K \frac{O-D_{O}}{R-D_{R}},
$$

where $I$ represents the corrected image, $R$ is the reference image from the reference plate, $O$ is the raw object image, $D_{O}$ and $D_{R}$ represent dark images taken with the corresponding integration times for the object and reference plate, respectively, and $K$ is a constant and usually is equal to the intensity averaged over the area of reference image. To demonstrate the necessity of correcting a raw image, Figure 2 shows the cross-section profiles along $X$ axis and $Y$ axis of a CCD image before and after flat field correction. It is noticed that the center of FOV detects much higher light intensity than the edges before correction. After the correction, it is clearly shown that the uniformity on the edges is much improved while the correction is not perfect.

For quantitative comparison, we calculated maximal relative deviations between the central intensity and the intensity at the edge of the images for both the raw and corrected images, using the following expression:

$$
\text { relative deviation }=\frac{\text { central intensity }}{\text { intensity at the edge }},
$$

The estimated maximal values of relative deviations were $40 \%$ and $10 \%$ for the raw image and corrected image, respectively. In most of the image regions, such deviations in the corrected images were less than $10 \%$.

2.3. Stability and Linearity of the CCD Camera System. To check the stability of the CCD camera system, repeated measurements were taken at different times. We utilized a $1 \%$ Intralipid solution (Baxter Healthcare Corporation, Deerfield, IL) as a testing medium and took optical readings as the baseline images $\left(I_{B}\right)$ at time " 0 ", followed by other images $\left(I_{M}\right)$ taken 1 minute, 5 minutes, 10 minutes, 30 minutes, 1 hour, and 1 day after the baseline measurement using the same experimental conditions (i.e., the same integration time). Each of the images was calibrated or corrected with the flat field correction and averaged over all the pixels per image to obtain an averaged light intensity for each respective image. Then, a ratio between the averaged intensity from each set of $I_{M}$ and the averaged intensity from $I_{B}$ (the baseline image) was obtained for all cases, 


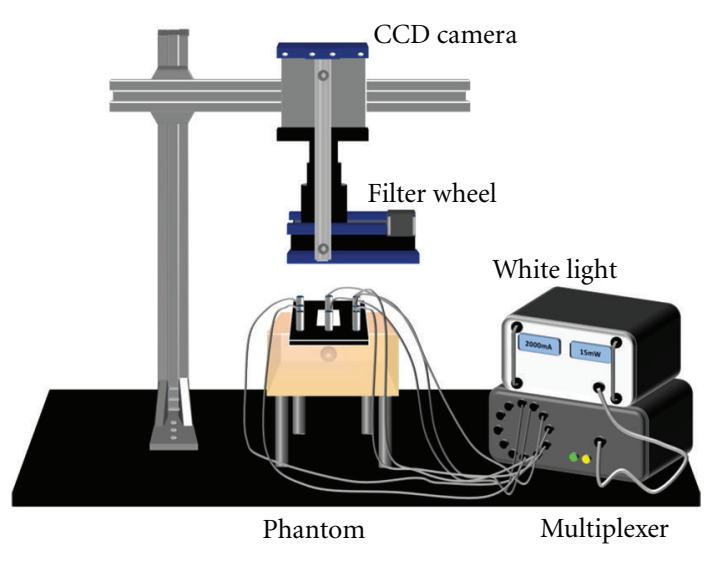

(a)

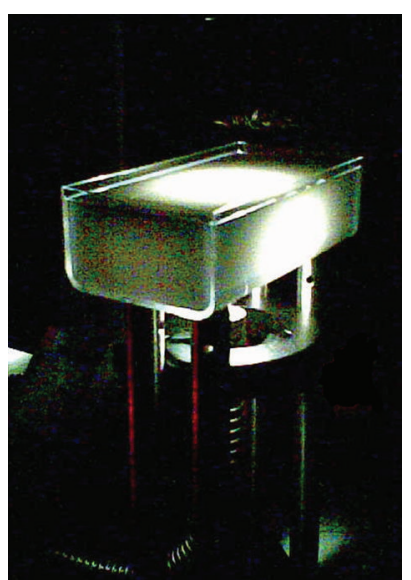

(b)

FIgURE 1: (a) Schematic diagram of a CCD-camera-based DOT system for laboratory measurement; (b) a tissue phantom which was sitting on the phantom supporter with the illumination light coming from the bottom.

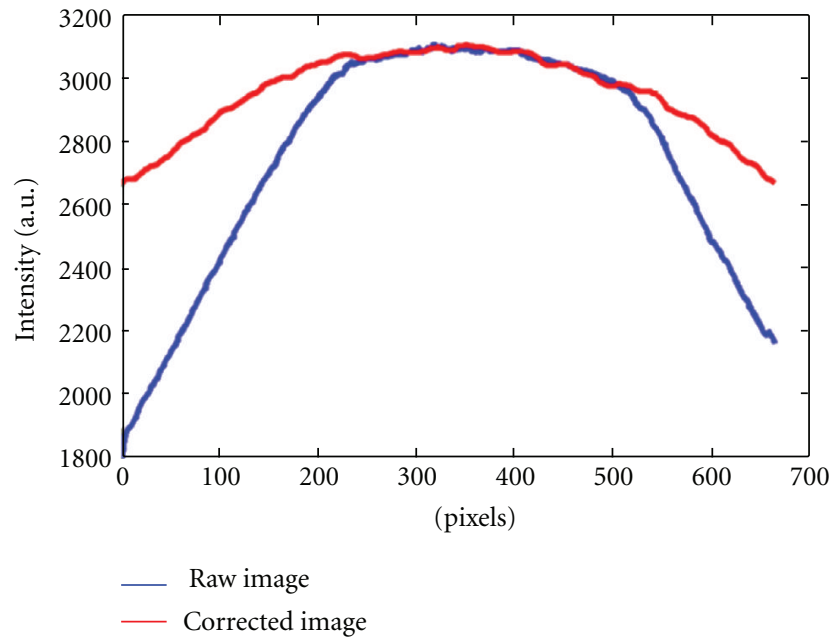

(a)

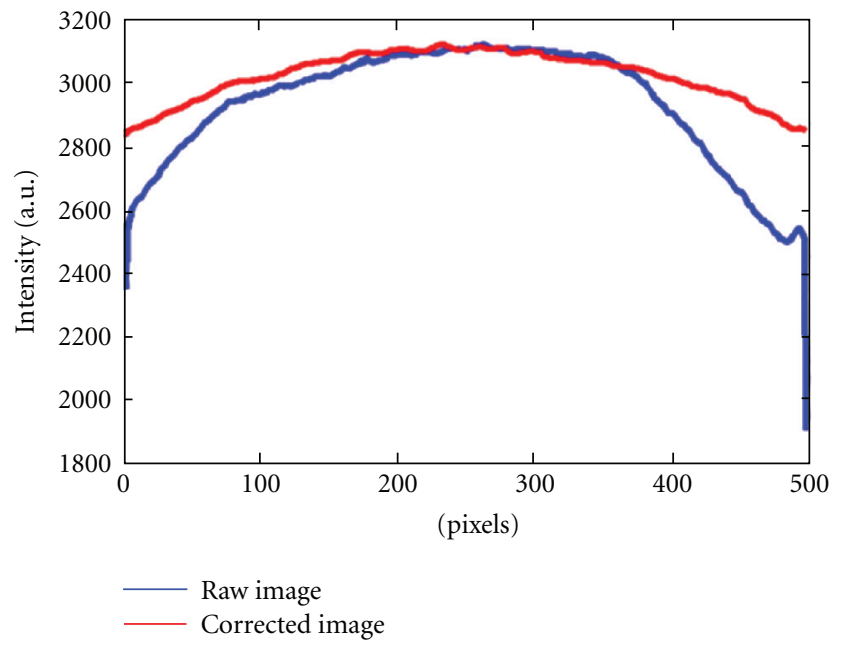

(b)

FIgURE 2: The cross-section profile of intensity from the raw and corrected image along (a) $X$ axis and (b) $Y$ axis.

as shown in Figure 3(a). It is very clear that the image intensities taken at different time intervals are fluctuated less than $1 \%$ of that of the baseline image, confirming good stability of the CCD camera system. Similar experiments were repeated using a $1.5 \%$ Intralipid solution to increase the light scattering property of the testing medium. The results are very consistent with the results from $1 \%$ Intralipid solution, demonstrating that a CCD camera has high stability with a fluctuation of intensity less than $1 \%$.

In addition, we checked whether the intensity of light source could affect the results. We took numerous images using the same Intralipid concentration, but with different intensities of the light source. The averaged intensity of an image taken at light source level 5 was selected as the baseline, $I_{B}$; the ratios between the averaged intensities from other images, $I_{M}$, with different light source levels to the chosen baseline, $I_{M} / I_{B}$, are shown in Figure $3(\mathrm{~b})$. This set of tests confirms that the level of the light source does not significantly affect the relative changes of our CCD camera output. Namely, the camera provides stable output images, independent of variable light levels.

Furthermore, to test the linearity of the CCD camera system, we performed two experiments: (1) one was to determine the image intensity changes versus absorption changes within the testing medium, and (2) the other one was to obtain the relationship between the image intensity measured versus changes in light scattering property within the testing medium. The tissue-mimic Intralipid solution was held in an acrylic box, which had a dimension of $10 \mathrm{~cm} \times$ $5 \mathrm{~cm} \times 3 \mathrm{~cm}$ (length $\times$ width $\times$ height). The acrylic box was wrapped by black tape except the top side of the box to prevent light from passing through the box wall.

For absorption changes, the experiments were performed using $1 \%$ Intralipid solutions with addition of $10 \mu \mathrm{L}, 20 \mu \mathrm{L}$, 


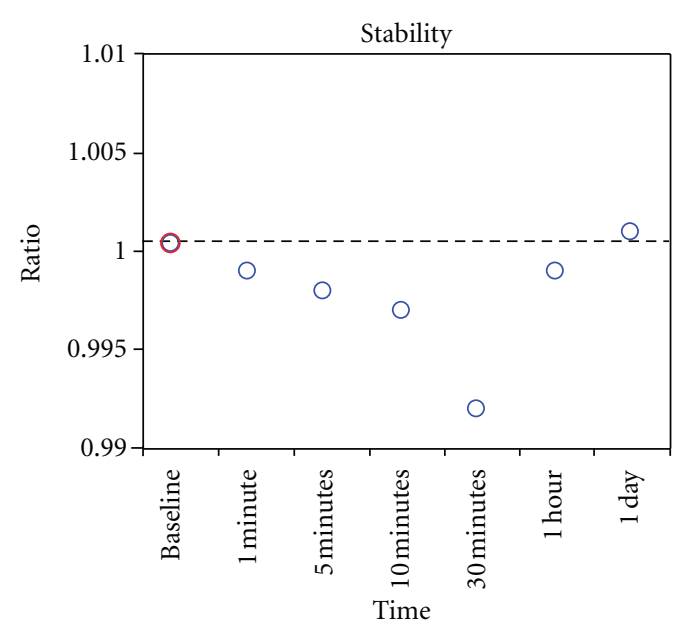

(a)

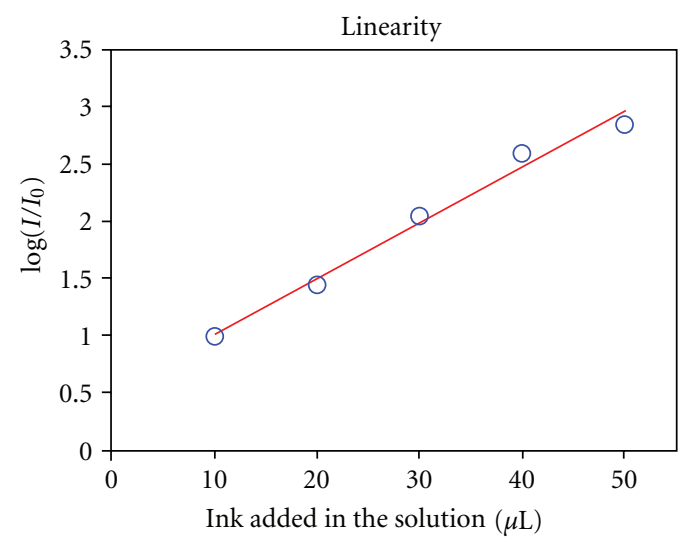

(c)

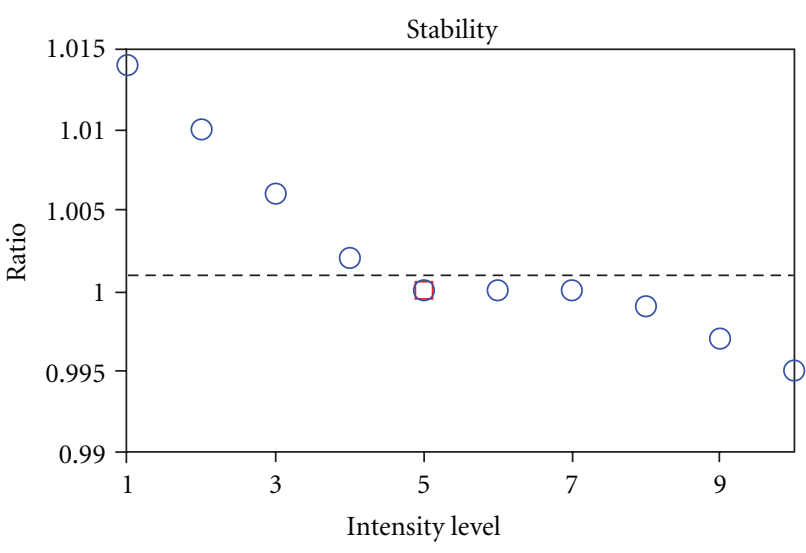

(b)

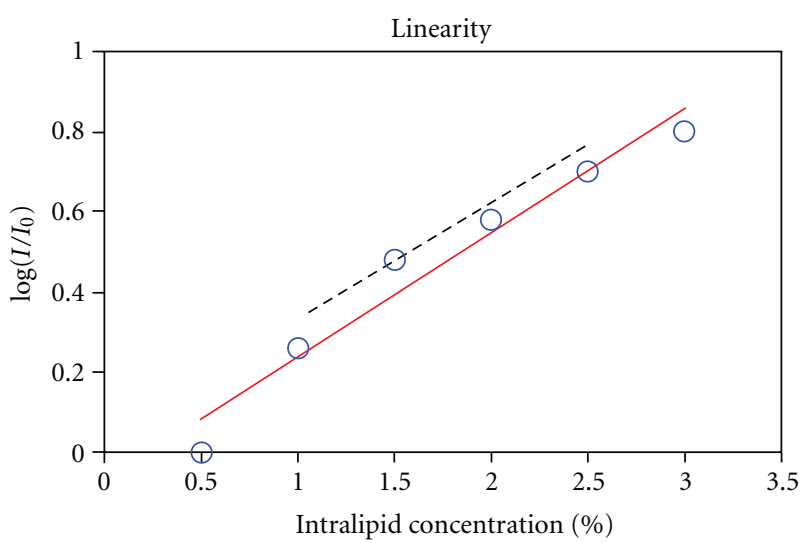

(d)

FIgURE 3: Ratio of the intensities $\left(I_{M} / I_{B}\right)$ for a $1 \%$ Intralipid solution (a) at different times of measurement and (b) with different light intensities. The relationships between $\log \left(I_{0} / I\right)$ and (c) the volume of ink added into the testing solution for a $1 \%$ Intralipid solution, and (d) the concentration of Intralipid. Dashed line in (d) shows the linear relationship within Intralipid concentrations between $1 \%$ and $2.5 \%$.

$30 \mu \mathrm{L}, 40 \mu \mathrm{L}$, and $50 \mu \mathrm{L}$ of diluted ink. The CCD camera readings were taken throughout these Intralipid solutions, using the same integration time. We further plotted values of $\log \left(I_{0} / I\right)$ versus the volume of the ink added in the solution, where $I_{0}$ and $I$ represented the averaged image intensities taken from the Intralipid solutions without ink and with different concentrations of ink. As seen in Figure 3(c), the data can be fitted well by a linear line. A similar behavior was seen for the $1.5 \%$ Intralipid case, confirming that a CCD camera has high-quality performance in its linearity with respect to light absorption.

For light scattering changes, the experiments were taken from multiple Intralipid solutions with concentrations of $0.5 \%, 1 \%, 1.5 \%, 2 \%, 2.5 \%$, and $3 \%$ (using the same integration time). A plot of $\log \left(I_{0} / I\right)$ versus concentration of Intralipid is given in Figure 3(d), where $I_{0}$ and $I$ represent the averaged image intensities taken from the Intralipid solutions at $0.5 \%$ and other concentrations. A good linear relationship is seen when Intralipid concentrations are between $1 \%$ and $2.5 \%$ (shown by the dashed line in Figure $3(\mathrm{~d})$ ). While changes of image intensity due to light scattering may nonlinearly depend on the Intralipid concentration [21], such non-linearity is relatively small and can be nonsignificant within the scattering property range for biological tissues $[22,23]$. Thus, the optical density measured from the CCD camera is linearly proportional to the light scattering changes, at the first-order approximation.

2.4. Optode Geometry for CCD-Camera-Based DOT. Instrument noise may appear during the measurements with a CCD camera that serves as multiple detectors; dark noise and nonuniformity of CCD chip sensitivity are two noise sources. Another problem often encountered is associated with the limited dynamic range of a CCD camera: when an individual pixel of the CCD array reaches its maximal intensity, the individual pixel well is occupied by electrons which can also overflow into the neighboring pixel wells. Such an overspill of electrons thereby distorts the actual signals not only at the saturated pixel but also at several adjacent pixels. In order to minimize the problem of signal saturations, the source fibers in this study were arranged around the FOV of the CCD camera, as shown in Figure 4. Such geometry can also be utilized while keeping the source fibers within the FOV. 


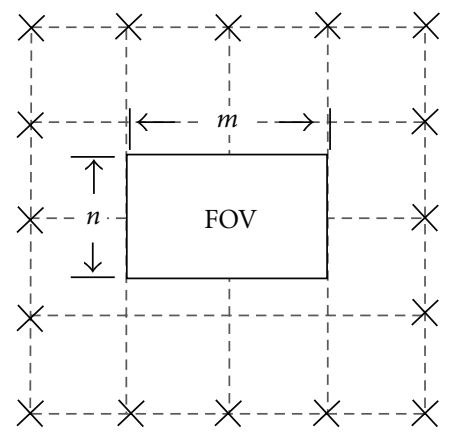

FIGURE 4: Optode geometry for CCD-camera-based DOT system. The dimension of FOV was $m \times n$ in $\mathrm{mm}^{2}$. $X$ represents possible locations of source fibers.

As shown in Figure 4, the dimension of FOV of the CCD camera was $m \times n \mathrm{~mm}^{2}$. Instead of using physical detectors, virtual detectors were used in this noncontact CCD camera imaging system. Virtual detectors refer to groups of CCD pixels combined within FOV of the CCD camera. The signalto-noise ratio (SNR) at selected locations can be enhanced by averaging the signals of all pixels selected. For example, if we selected $10 \times 10$ pixels to be binned as a virtual detector, the entire FOV would have a total number of $\sim 50 \times 66$ virtual detectors for the DOT measurements. This implies that a CCD-camera-based DOT system may serve as a highdensity imaging device with an easy-to-change detector array by grouping the pixels in different ways as detectors.

\subsection{Theory of Image Reconstruction}

2.5.1. Conventional Image Reconstruction in DOT. The photon propagation in a highly scattering medium follows the diffusion equation, and the analytic solution of the diffusion equation can be utilized to study optical signal changes due to absorption perturbation, as given by

$$
\begin{aligned}
\Delta \mathrm{OD} & =-\log \left(\frac{\Phi_{\text {pert }}}{\Phi_{0}}\right)=-\frac{1}{2.3} \ln \left(\frac{\Phi_{\text {pert }}}{\Phi_{0}}\right) \\
& =\frac{1}{2.3} \int \Delta \mu_{a}(r) L(r) d r,
\end{aligned}
$$

where $\Delta \mathrm{OD}$ is the change in optical density. $\Phi_{0}$ is the photon fluence in a semi-infinite, homogeneous medium, and $\Phi_{\text {pert }}$ is the photon fluence with absorbers embedded. $L$ is the effective pathlength of light through the tissue experiencing the absorption change. While (3) is known as the modified Beer-Lambert law, it can be generalized in a matrix form and written as $\mathbf{y}=\mathbf{A x}$, where matrix $\mathbf{y}$ represents measured changes in optical density from all the measurements, matrix $\mathbf{x}$ corresponds to a volumetric vector of the unknown perturbation in absorption coefficient in the volume under study, and matrix A describes the sensitivity of measurements to the change in absorption within each voxel in the medium.

However, solving $\mathbf{y}=\mathbf{A x}$ is nontrivial, involving an underdetermined, ill-posed inverse problem because the number of measurements is much fewer than the number of unknowns (i.e., voxels) to be reconstructed. In the current research field, to reconstruct a DOT image, the Tikhonov regularization [24] method has been employed; an image of $\mathbf{x}$ can be given by

$$
\hat{x}=A^{T}\left(A A^{T}+\alpha S_{\max } I\right)^{-1} y,
$$

where $\hat{x}$ is the reconstructed image of absorption perturbation; $I$ is the identity matrix; $S_{\max }$ is the maximal singular value of matrix $A A^{T}$, and $\alpha$ is the regularization parameter.

2.5.2. Depth Compensation Algorithm. Because of the severe attenuation in light intensity as increase of propagation depth, the measurement sensitivity of DOT in deep tissue is significantly lower than that in superficial tissue. In order to overcome this problem, a new approach of DCA has been recently developed by introducing a weighted matrix $\mathbf{M}$, which has a pseudo-exponential magnitude with depth to counterbalance the loss of sensitivity of $\mathbf{A}$ in deep layers. The weighted matrix $M$ is formed as

$$
M=\operatorname{diag}\left(M\left(A_{L}\right), M\left(A_{L-1}\right), \ldots, M\left(A_{2}\right), M\left(A_{1}\right)\right)^{\gamma},
$$

where $M\left(A_{i}\right)$ represents the maximum singular values for measurement sensitivities within the particular layer $i$ which is decomposed from forward matrix $A ; \gamma$ is an adjustable power and can be varied between 0 and 3 . The detailed derivation and validation of DCA can be found in references $[18,19]$. The key component in DCA is the formation of a transformation matrix $\mathbf{A}^{\#}$, as defined by $\mathbf{A}^{\#}=\mathbf{A M}$. Then $\mathbf{A}^{\#}$ is used to replace the original sensitivity matrix, $\mathbf{A}$, in (4). The reconstructed image to be obtained using DCA can be written as follows:

$$
\hat{x}_{\mathrm{DCA}}=(A M)^{T}\left(A M(A M)^{T}+\alpha S_{\max }^{\prime} I\right)^{-1} y,
$$

where $S_{\max }^{\prime}$ is the maximum eigenvalue of $A M(A M)^{T}$. Notice that $\hat{x}_{\mathrm{DCA}}$ in (6) is not equal to $\hat{x}$ in (4) since matrix $M$ is empirically introduced. It is known now that (6) yields minimum error in depth localization for reconstructed NIRS images, while the trade-off of DCA is an inaccurate quantification for absorption perturbation.

As demonstrated in our recent publications $[18,19]$, the power of depth compensation is controlled by the exponent parameter of $\gamma$ in $M$. The larger the $\gamma$ value is, the larger the weight is to compensate the measurement sensitivity toward a deeper layer, and vice versa. An inappropriate selection of $\gamma$ could lead to overcompensation and give rise to inaccurate results. Our previous studies have suggested an optimal range of $\gamma$ to be 1.0-1.6 for accurate depth localization using a fiber-based DOT system. In this study, we need to select and confirm a suitable range of $\gamma$ for a CCD-camera-based DOT system. 


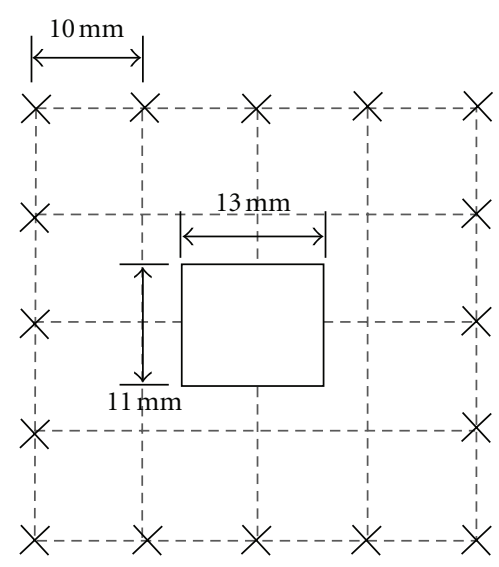

(a)

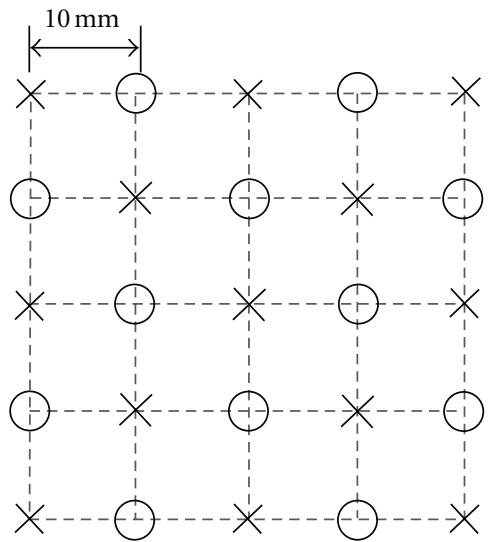

(b)

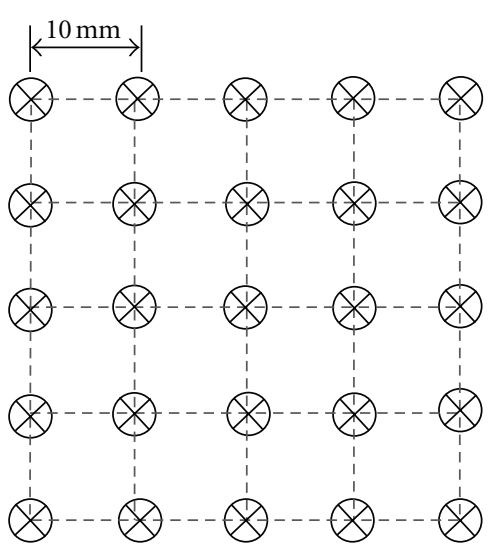

(c)

Figure 5: Scheme of optode geometries used in the simulation study. (a) to (c) are corresponded to G-I to G-III. Notice that the dimension of all dashed unit squares is $10 \times 10 \mathrm{~mm}^{2}$. (x: source fibers; o: detector fibers; $\otimes$ : bifurcated fibers).

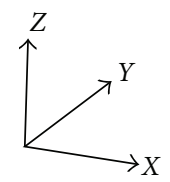

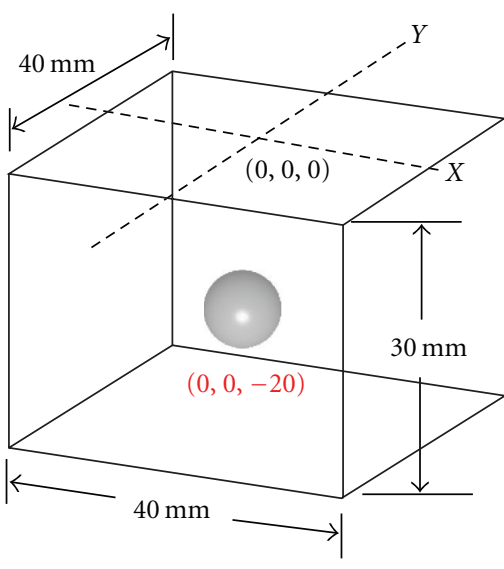

(a)

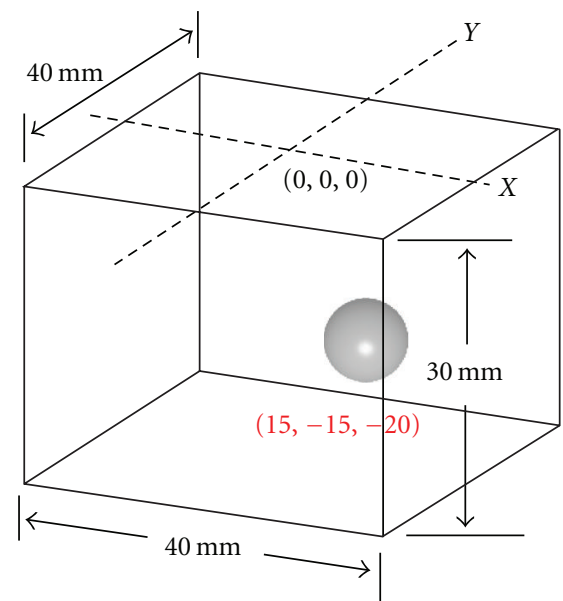

(b)

Figure 6: Schematic diagram of setups used in simulation study. (a) Setup I: the inclusion was located right below the center of measurement plane. (b) Setup II: the inclusion was located close to the right side corner of the measurement volume.

\section{Results}

3.1. Computer Simulations. To evaluate reconstruction performances using the source-camera geometry given in Section 2.4 (also replotted in Figure 5(a) and denoted as GI), we performed computer simulations and compared the results with those that were derived using the source-detector geometries commonly seen in the fiber-based DOT system (shown in Figures 5(b) and 5(c)). Several sets of simulations were carried out under the conditions of (1) using different numbers of sources and virtual detectors, (2) without and (3) with utilization of DCA.

As shown in Figure 5(a), sixteen sources were used in GI. In terms of the number of virtual detectors, we defined the dimension of FOV to be $13 \times 11 \mathrm{~mm}^{2}$ and the dimension of each virtual detector to be $1 \mathrm{~mm}^{2}$. Therefore, there were a total of $13 \times 11=143$ virtual detectors used in the simulations. The geometry shown in Figure 5(b) (G-II) is commonly used optode geometry with sources and detectors being alternately placed. The geometry given in Figure 5(c) (G-III) exhibits the optode setup with bifurcated fibers, that is, each fiber can serve as a source and detector.

The reconstruction performances were evaluated by two parameters: (1) the position error ( $\mathrm{PE}$ ) and (2) contrast-tonoise ratio (CNR) [25]. PE is the distance from the center of actual object to the center of the reconstructed object. The reconstructed object was defined by the pixels with values above half of the maximum value. CNR indicates whether the reconstructed object can be clearly distinguished from the background. A reconstructed image was divided into two regions: volume of interest (VOI) and volume of background (VOB). VOI was defined by the location and 


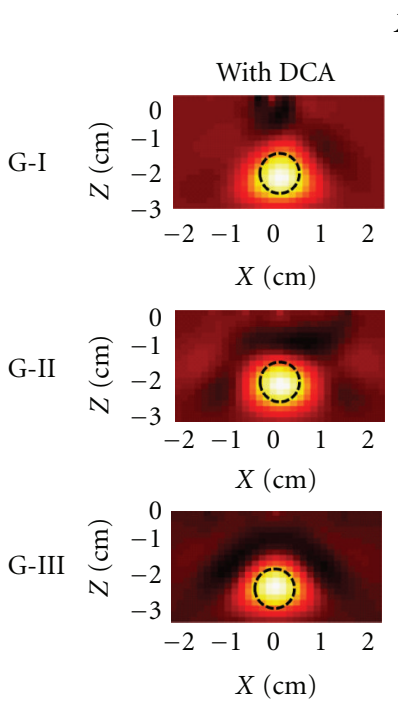

(a)
$X-Z$ plane
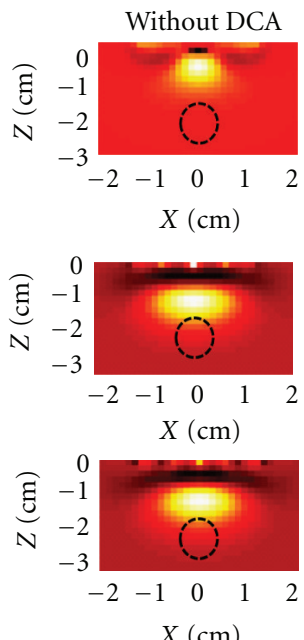

(b)
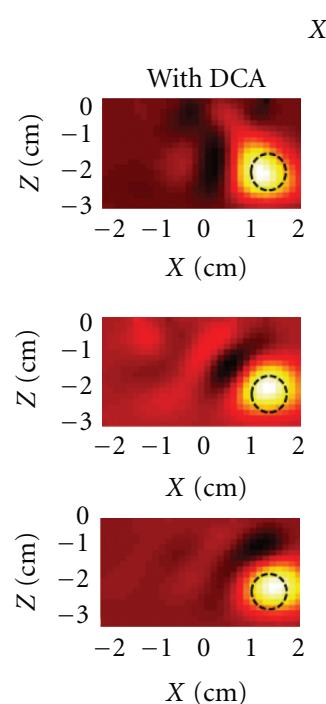

(c)
$X-Z$ plane
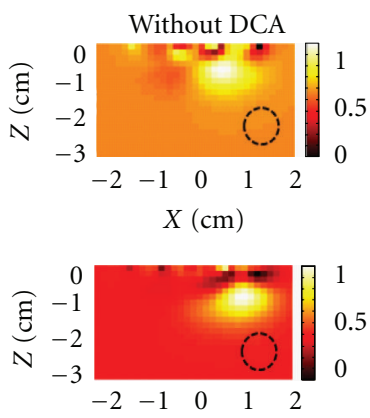

$X(\mathrm{~cm})$

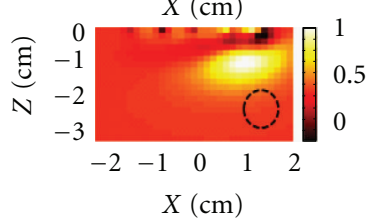

(d)

FIgure 7: Reconstructed DOT images of a single object in $X-Z$ plane: (a) and (c) with DCA ( $\gamma=1.3$ ), and (b) and (d) without DCA. Top-to-bottom rows were obtained from G-I to G-III (Figure 5).

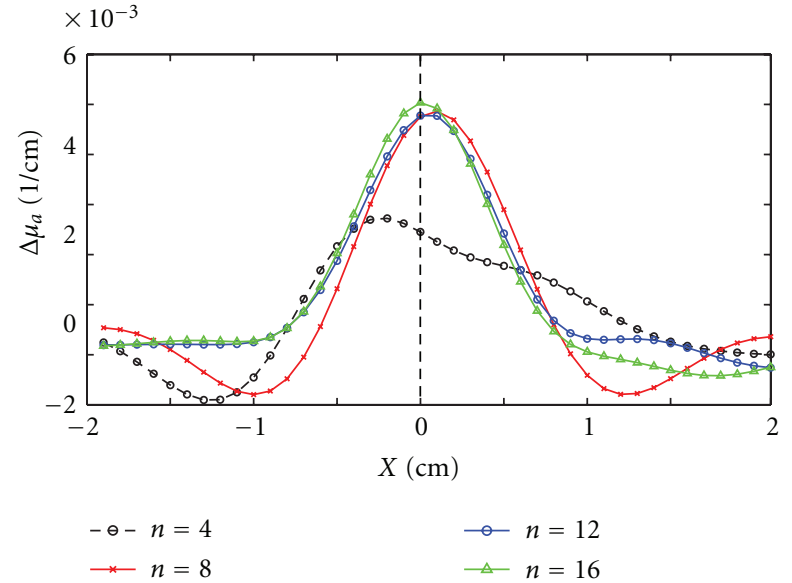

(a)

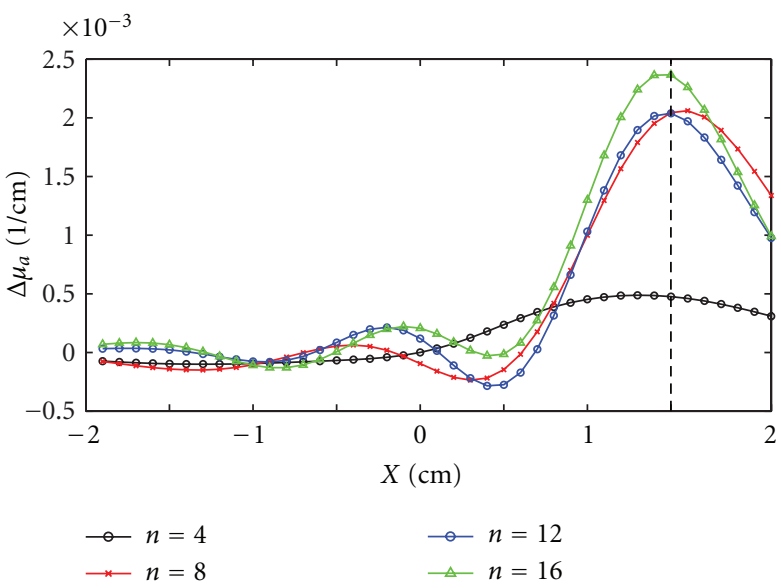

(b)

Figure 8: Profiles of reconstructed $\Delta \mu_{a}$ values along $x$-axis for (a) experimental Setup I and (b) experimental Setup II. Dashed vertical lines represent the actual location of the inclusion in $x$-axis.

size of the reconstructed object. VOB was defined by the remaining volume of the image. CNR can be calculated by

$$
\mathrm{CNR}=\frac{\mu_{\mathrm{VOI}}-\mu_{\mathrm{VOB}}}{\left[w_{\mathrm{VOI}} \sigma_{\mathrm{VOI}}^{2}+w_{\mathrm{VOB}} \sigma_{\mathrm{VOB}}^{2}\right]^{1 / 2}},
$$

where $w_{\mathrm{VOI}}$ and $w_{\mathrm{VOB}}$ are the weight factors of the VOI and $\mathrm{VOB}$ relative to the entire volume (i.e., VOI or VOB divided by the entire volume), $\mu_{\mathrm{VOI}}$ and $\mu_{\mathrm{VOB}}$ are the mean values of object and background volumes in a $3 \mathrm{D}$ reconstructed image, and $\sigma_{\mathrm{VOI}}$ and $\sigma_{\mathrm{VOB}}$ are the respective standard deviations.

3.1.1. Image Reconstruction of Embedded Objects. In the sim ulation, we considered a homogeneous semi-infinite medium with a volume of $40 \times 40 \times 30 \mathrm{~mm}^{3}$ (Figure 6). The origin of the coordinates was set at the center of top surface, as marked in the figure; this surface was also chosen to be the measurement surface in the simulation. As commonly used, a set of absorption and reduced scattering coefficients of $\mu_{a}=0.1 \mathrm{~cm}^{-1}$ and $\mu_{s}^{\prime}=10 \mathrm{~cm}^{-1}$ were selected to simulate the background tissue.

Two different setups were utilized to evaluate the performances of optode geometries: Setup 1 is shown in Figure 6(a), where a spherical absorber with an $8 \mathrm{~mm}$ diameter was placed at $(X, Y, Z)=(0,0,-20)$ (in $\mathrm{mm}$ ), with a $3: 1$ contrast ratio in $\mu_{a}$ relative to the background $\mu_{a}$. By looking at the optode geometry of the CCD-camerabased DOT imaging system, it is noticed that there are fewer overlapping measurements near four corners of FOV 


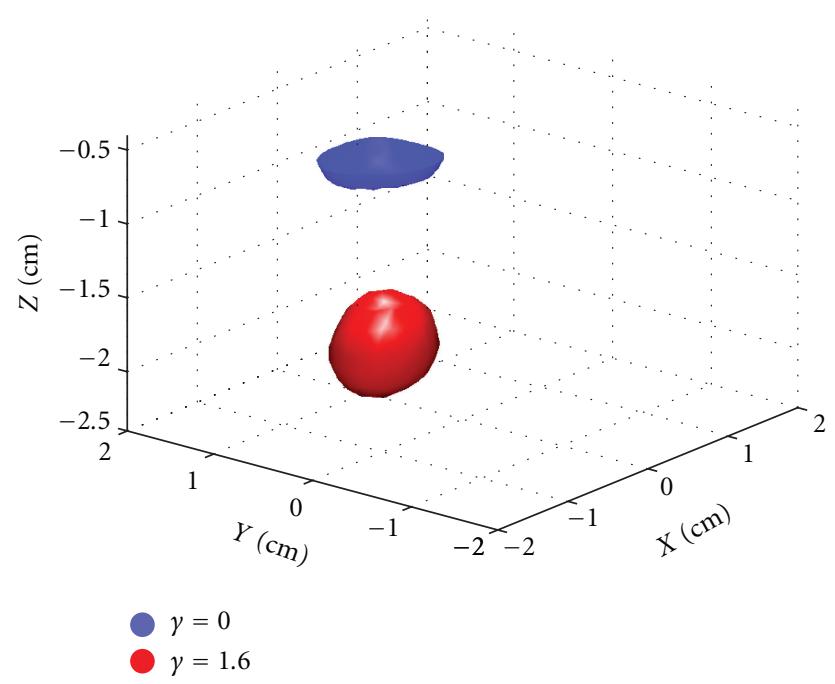

(a)

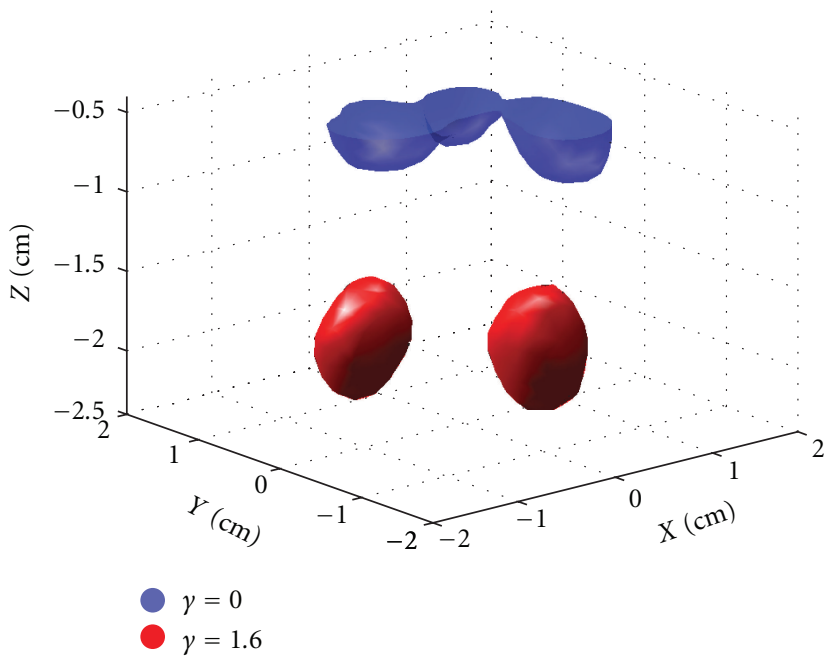

(b)

FIGURE 9: Three dimensional plots of reconstructed images for (a) one inclusion with DCA $(\gamma=1.6)$ and without DCA (i.e., $\gamma=0)$, and (b) two inclusions with DCA $(\gamma=1.6)$ and without DCA $(\gamma=0)$. Voxels with the reconstructed absorption values above $80 \%$ of the maximum value are selected as reconstructed objects.

of the camera, as compared to the center of FOV. This is because more overlapping measurements occur near the center of FOV when we pair most of the virtual detectors with given light sources in the current source-camera setup. It has also shown that a higher measurement density does not necessarily improve the spatial resolution significantly [26]. Thus, to evaluate and compare the effect of the number of overlapping measurements at the four corners, in Setup 2, one spherical absorber was placed near the right corner of the medium with respect to Setup 1, as shown in Figure 6(b). A random Gaussian noise of $1 \%$ in amplitude was added to the simulated measurement data. DCA was employed with a compensation power of $\gamma=1.3$.

The reconstructed images in the $X-Z$ plane from both setups are shown in Figure 7: Figures $7(\mathrm{a})$ and $7(\mathrm{~b})$ are obtained from Setup 1 with the absorber placed at the center of measurement plane, while Figures $7(\mathrm{c})$ and $7(\mathrm{~d})$ result from Setup 2. Also, the three rows from top to bottom in the figure correspond to the fiber-camera or fiber-fiber geometry of G-I to G-III, respectively. Figures 7(b) and 7(d) clearly show that the reconstructed images for all geometries are pulled towards the superficial surface along $Z$ direction when DCA is not used or $\gamma=0$. After DCA was employed with $\gamma=1.3$, the reconstructed object was reconstructed at the expected depth (Figures 7(a) and 7(c)). By comparing the reconstructed images using the three geometries of G-I to G-III, we can see that the overall qualities of DOT images are all similar. However, in Figure 7(c), it is noticed that the DOT images with G-II and G-III exhibit a relatively better shape for the reconstructed objects, while G-I leads to a little distorted profile (tilted to the center) when the absorber was close to the edge of the imaging medium.

To further quantify the comparison among the three geometries, we have calculated respective values of CNR and PE from each geometry (Figure 5) and for two simulation setups (Figure 6), and the corresponding values are listed in Table 1. It is clearly seen that there are significant improvements of PE values with DCA, as we expected, for both setups, while the overall PE values in Setup 1 are better than those in Setup 2. Next, we focus on only the data with DCA: by close inspection on G-I to G-III for Setup 1, we observe that CNR values from all three optode geometries are not greatly different although G-III has a little higher CNR. A similar trend can be seen for Setup 2. Regarding PE values obtained only with DCA, no noticeable difference appears for all three geometries (less than $1 \mathrm{~mm}$ ) in either of the setups.

Overall, the computer simulation results confirm that the geometry of G-I, which can be utilized as a CCD-camerabased DOT system, is able to deliver excellent DOT images at the same quality level as the other two fiber-based DOT systems do. Given the simplicity, low cost, and portability of such a system, it may be preferred in preclinical, small animal studies.

3.1.2. Optimization of the Number of Sources. It is common that the space available in preclinical studies around animals is restricted or limited to allow for many optodes. Thus, we next further investigate a possible optimal number of optodes that can still permit a reasonably good DOT image. In geometry G-I, only source fibers need to be arranged around FOV of the CCD camera. Therefore, several computer simulations were performed based on both setups (Figure 6) used in Section 3.1.1 in order to optimize the numbers of sources and virtual detectors. Specifically, the light sources were placed symmetrically around the origin to prevent the distortion of reconstructed images.

Figure 8 shows several cross-section profiles of the reconstructed $\Delta \mu_{a}$ values along $x$-axis with different numbers of sources while the absorber was placed at center (Figure 8(a)) 
TABLE 1: Summary of the CNR and PE values for three geometries.

\begin{tabular}{lcccccccccccr}
\hline & \multicolumn{4}{c}{ Setup 1 (absorber at center) } & \multicolumn{4}{c}{ G-II } & \multicolumn{4}{c}{ Getup 2 (absorber on side) } \\
& \multicolumn{2}{c}{ G-I } & \multicolumn{2}{c}{ G-II } & \multicolumn{2}{c}{ G-II } & \multicolumn{2}{c}{ G-III } \\
& $W$ & $W / O$ & $W$ & $W / O$ & $W$ & $W / O$ & $W$ & $W / O$ & $W$ & $W / O$ & $W$ & $W / O$ \\
\hline $\mathrm{CNR}$ & 5.66 & 4.39 & 5.65 & 4.12 & 5.73 & 4.58 & 5.45 & 5.46 & 5.61 & 5.62 & 6.47 & 4.72 \\
$\mathrm{PE}(\mathrm{cm})$ & 0.20 & 0.82 & 0.20 & 0.75 & 0.20 & 0.72 & 0.36 & 0.81 & 0.35 & 0.79 & 0.35 & 0.78 \\
\hline
\end{tabular}

*W: with DCA; W/O: without DCA.

TABLE 2: Summary of the CNR, PE, and CPU time for different numbers of sources.

\begin{tabular}{lcccccccc}
\hline & & \multicolumn{2}{c}{ Setup I (absorber at center) } & \multicolumn{4}{c}{ Setup II (absorber on side) } \\
Numbers of source & $\mathbf{4}$ & $\mathbf{8}$ & $\mathbf{1 2}$ & $\mathbf{1 6}$ & $\mathbf{4}$ & $\mathbf{8}$ & $\mathbf{1 2}$ & $\mathbf{1 6}$ \\
\hline CNR & 4.27 & 5.66 & 5.67 & 5.83 & 1.24 & 4.42 & 4.51 \\
PE $(\mathrm{cm})$ & 0.41 & 0.20 & 0.20 & 0.20 & 0.88 & 0.35 & 0.32 \\
CPU time $(\mathrm{sec})$ & 27.1 & 102.1 & 209.4 & 289.2 & 27.1 & 102.1 & 209.4 & 289.2 \\
\hline
\end{tabular}

and close to the edge of medium (Figure $8(\mathrm{~b})$ ). It is clear from this figure that an increase in number of the sources can lead to an enhanced reconstructed contrast, and thus resulting in a clearer DOT image. However, no substantial difference is observed in absorption contrast while the number of sources is increased from 8 to 12 and further to 16 in both cases.

More quantitatively, we have calculated CNR and PE values for all four cases with the number of sources of $n=4$, 8,12 , and 16 . The respective values are given in Table 2. It shows unambiguously that no difference in PE and CNR appears when $n$ has changed from 8 to 16 . However, the computational time for $n=16$ is $\sim 2.5$ times longer than that for $n=8$. Hence, for practical use, we would suggest to utilize eight sources instead of twelve or more, which offer the same quality of DOT images but have less complexity of a DOT system.

3.2. Phantom Experiments and Results. Besides the computational investigation given above, we have also performed laboratory phantom studies to confirm what we found. A $1 \%$ Intralipid solution was used to generate the liquid tissue phantom (Figure 1(b)) with background optical properties of $\mu_{a}=0.1 \mathrm{~cm}^{-1}$ and $\mu_{s}^{\prime}=10 \mathrm{~cm}^{-1}$. A spherical gelatin inclusion of $8 \mathrm{~mm}$ in diameter was embedded $20 \mathrm{~mm}$ below the liquid surface, with a $2.5: 1$ contrast ratio in absorption between the inclusion and background. A broad-band white light source (Illumination Technologies, Inc. Model 3900, quartz-tungsten halogen lamp) was used, and a 12-bit CCD camera (SamBa Q34 with Navitar Zoom 7000 lens) was employed as the detector. The FOV of the camera was $13 \times 11 \mathrm{~mm}^{2}$, the same dimension as used in the simulations. The total FOV was divided into $143(13 \times 11)$ virtual detectors, meaning that each virtual detector had $38 \times 38$ pixels. In this study, 8 light sources were connected to and controlled by a multiplexer (Avantes Inc. Multiplex Channels $1 \times 16$ ), as shown in Figure 1(a). A total of $8 \times 143$ source-detector (virtual) pairs were pooled to perform DOT image reconstructions with utilization of DCA. The total volumetric imaging space was $40 \times 40 \times 30 \mathrm{~mm}^{3}$. Moreover, another similar experiment was repeated with two identical inclusions embedded at $20 \mathrm{~mm}$ depth.

3D reconstructed images are shown in Figures 9(a) and 9 (b) for the phantom experiments described above. To obtain $3 \mathrm{D}$ plots, we have selected the reconstructed absorption values at $80 \%$ of its maximum as a volume threshold in order to form the imaged object. From both Figures 9(a) and 9(b), it is clear that the embedded objects can be successfully reconstructed at a relatively accurate depth when DCA is applied. However, because of the diffuse nature of light in tissues, the volume ratio between the reconstructed and real object is around $2: 1$, which also depends on the threshold value selected and the depth of the embedded inclusion.

\section{Conclusion}

In this study, we present the feasibility of using a CCDcamera-based system combined with our newly developed DCA to form volumetric diffuse optical tomography. We performed both computer simulations and laboratory phantom experiments to confirm our findings. Although only a 12-bit CCD camera was utilized in our experiment setup, the optical signals from $20 \mathrm{~mm}$ depth could still be detected by grouping several pixels from the CCD-camera readings as virtual detectors. Overall, we are confident to state that because of its simplicity, low cost, and portability of such a system, a CCD-camera-based DOT may become a highly appropriated imaging tool to be applied in preclinical, small animal studies. For example, this approach may present a great promise for 3D localization of an embedded anomaly tissue, such as a tumor embedded in a small animal.

For future work, in vivo animal measurements are planned for both brain imaging and tumor detection to further validate volumetric imaging by combining a CCDcamera-based DOT system with DCA. In addition, optimization of the source-detector geometry will be further investigated since different combinations of source-detector arrangement may result in different qualities of reconstructed images. 


\section{Authors' Contribution}

The first two authors contributed equally to the paper.

\section{Acknowledgments}

This work was supported in part by NINDS from the NIH (5R33NS052850-04). The authors also acknowledge the PMI DOT imaging toolbox.

\section{References}

[1] N. Iftimia and H. Jiang, "Quantitative optical image reconstruction of turbid media by use of direct-current measurements," Applied Optics, vol. 39, no. 28, pp. 5256-5261, 2000.

[2] A. M. Siegel, J. J. A. Marota, and D. A. Boas, "Design and evaluation of a continuous-wave diffuse optical tomography system," Optics Express, vol. 4, no. 8, pp. 287-298, 1999.

[3] D. A. Boas, A. M. Dale, and M. A. Franceschini, "Diffuse optical imaging of brain activation: approaches to optimizing image sensitivity, resolution, and accuracy," NeuroImage, vol. 23, no. 1, pp. S275-S288, 2004.

[4] B. R. White, A. Z. Snyder, A. L. Cohen et al., "Resting-state functional connectivity in the human brain revealed with diffuse optical tomography," NeuroImage, vol. 47, no. 1, pp. 148-156, 2009.

[5] A. Custo, D. A. Boas, D. Tsuzuki et al., "Anatomical atlasguided diffuse optical tomography of brain activation," $\mathrm{Neu}$ roImage, vol. 49, no. 1, pp. 561-567, 2010.

[6] D. R. Leff, O. J. Warren, L. C. Enfield et al., "Diffuse optical imaging of the healthy and diseased breast: a systematic review," Breast Cancer Research and Treatment, vol. 108, no. 1, pp. 9-22, 2008.

[7] B. J. Tromberg, B. W. Pogue, K. D. Paulsen, A. G. Yodh, D. A. Boas, and A. E. Cerussi, "Assessing the future of diffuse optical imaging technologies for breast cancer management," Medical Physics, vol. 35, no. 6, pp. 2443-2451, 2008.

[8] G. Xu, D. Piao, C. H. Musgrove, C. F. Bunting, and H. Dehghani, "Trans-rectal ultrasound-coupled near-infrared optical tomography of the prostate-part I: simulation," Optics Express, vol. 16, no. 22, pp. 17484-17504, 2008.

[9] Z. Jiang, D. Piao, G. Xu et al., "Trans-rectal ultrasoundcoupled near-infrared optical tomography of the prostatepart II: experimental demonstration," Optics Express, vol. 16, no. 22, pp. 17505-17520, 2008.

[10] J. P. Sacha, M. D. Cockman, T. E. Dufresne, and D. P. Trokhan, "Quantification of regional fat volume in rat MRI," in Medical Imaging 2003: Physiology and Function: Methods, Systems, and Applications, vol. 5031 of Proceedings of SPIE, pp. 289-297, February 2003.

[11] A. Laib, O. Barou, L. Vico, M. H. Lafage-Proust, C. Alexandre, and P. Rügsegger, "3D micro-computed tomography of trabecular and cortical bone architecture with application to a rat model of immobilisation osteoporosis," Medical and Biological Engineering and Computing, vol. 38, no. 3, pp. 326-332, 2000.

[12] D. Sorger, M. Patt, P. Kumar et al., "[18F]Fluoroazomycinarabinofuranoside (18FAZA) and [18F]Fluoromisonidazole (18FMISO): a comparative study of their selective uptake in hypoxic cells and PET imaging in experimental rat tumors," Nuclear Medicine and Biology, vol. 30, no. 3, pp. 317-326, 2003.
[13] C. Vanhove, T. Lahoutte, M. Defrise, A. Bossuyt, and P. R. Franken, "Reproducibility of left ventricular volume and ejection fraction measurements in rat using pinhole gated SPECT," European Journal of Nuclear Medicine and Molecular Imaging, vol. 32, no. 2, pp. 211-220, 2005.

[14] J. P. Culver, T. Durduran, D. Furuya, C. Cheung, J. H. Greenberg, and A. G. Yodh, "Diffuse optical tomography of cerebral blood flow, oxygenation, and metabolism in rat during focal ischemia," Journal of Cerebral Blood Flow and Metabolism, vol. 23, no. 8, pp. 911-924, 2003.

[15] G. Gulsen, O. Birgul, M. B. Unlu, R. Shafiiha, and O. Nalcioglu, "Combined diffuse optical tomography (DOT) and MRI system for cancer imaging in small animals," Technology in Cancer Research and Treatment, vol. 5, no. 4, pp. 351-363, 2006.

[16] Q. Zhao, L. Ji, and T. Jiang, "Improving depth resolution of diffuse optical tomography with a layer-based sigmoid adjustment method," Optics Express, vol. 15, no. 7, pp. 40184029, 2007.

[17] B. W. Pogue, T. O. McBride, J. Prewitt, U. L. Österberg, and K. D. Paulsen, "Spatially variant regularization improves diffuse optical tomography," Applied Optics, vol. 38, no. 13, pp. 29502961, 1999.

[18] H. Niu, Z. Lin, F. Tian, S. Dhamne, and H. Liu, "Comprehensive investigation of three-dimensional diffuse optical tomography with depth compensation algorithm," Journal of Biomedical Optics, vol. 15, no. 4, article 046005, 2010.

[19] H. Niu, F. Tian, Z. Lin, and H. Liu, "Development of a compensation algorithm for accurate depth localization in diffuse optical tomography," Optics Letters, vol. 35, no. 3, pp. 429-431, 2010.

[20] S. B. Howell, Handbook of CCD Astronomy, 2000.

[21] S. C. Kanick, U. A. Gamm, M. Schouten, H. J. Sterenborg, D. J. Robinson, and A. Amelink, "Measurement of the reduced scattering coefficient of turbid media using single fiber reflectance spectroscopy: fiber diameter and phase function dependence," Biomedical Optics Express, vol. 2, pp. 1687-1702, 2011.

[22] M. Johns, C. A. Giller, D. C. German, and H. Liu, "Determination of reduced scattering coefficient of biological tissue from a needle-like probe," Optics Express, vol. 13, no. 13, pp. 48284842, 2005.

[23] G. Zonios and A. Dimou, "Modeling diffuse reflectance from semi-infinite turbid media: application to the study of skin optical properties," Optics Express, vol. 14, no. 19, pp. 86618674, 2006.

[24] S. R. Arridge, "Optical tomography in medical imaging," Inverse Problems, vol. 15, no. 2, pp. R41-R49, 1999.

[25] Q. Zhao, L. Ji, and T. Jiang, "Improving performance of reflectance diffuse optical imaging using a multicentered mode," Journal of Biomedical Optics, vol. 11, no. 6, Article ID 064019, 2006.

[26] F. Tian, G. Alexandrakis, and H. Liu, "Optimization of probe geometry for diffuse optical brain imaging based on measurement density and distribution," Applied Optics, vol. 48, no. 13, pp. 2496-2504, 2009. 

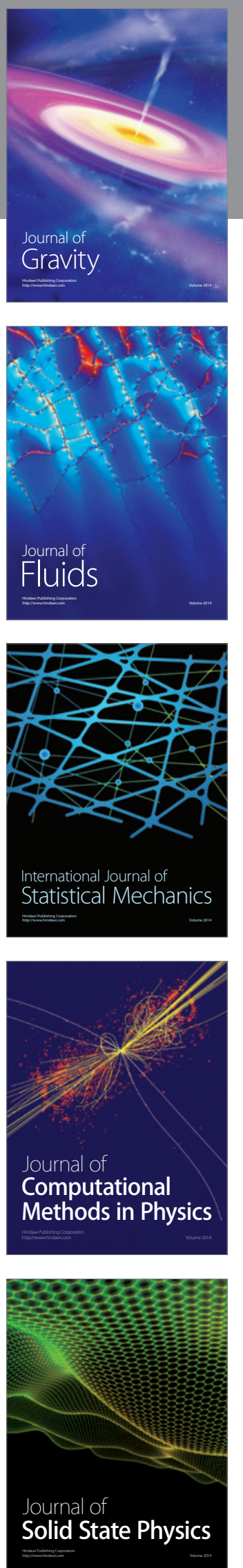

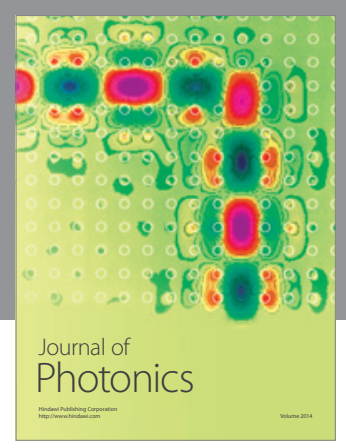

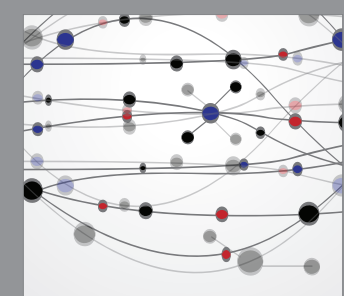

The Scientific World Journal
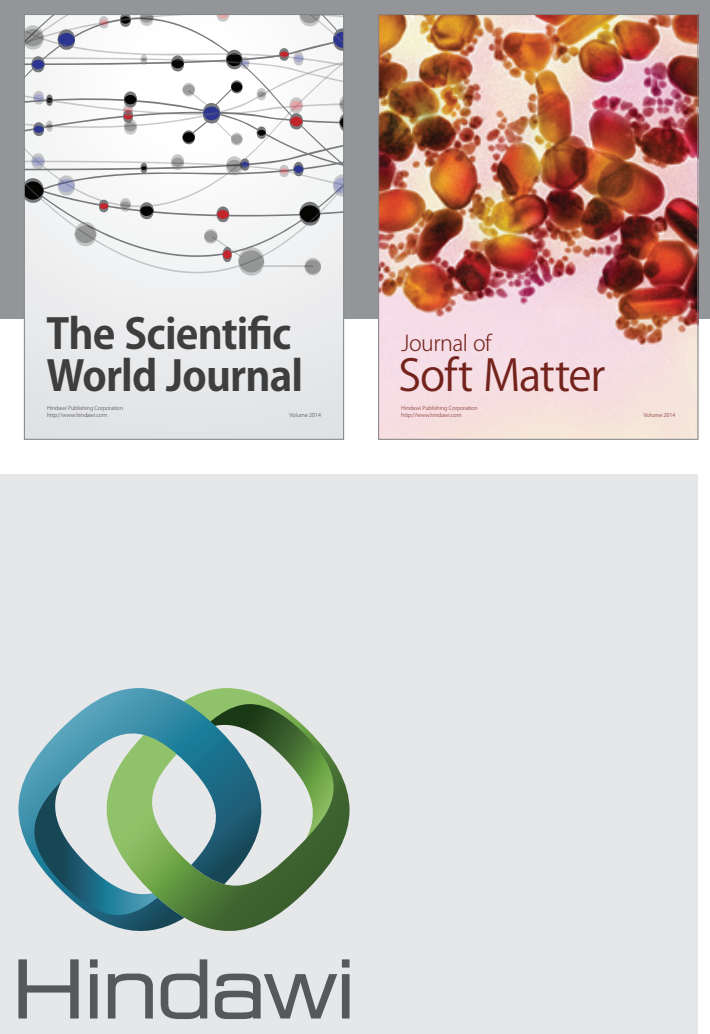

Submit your manuscripts at

http://www.hindawi.com
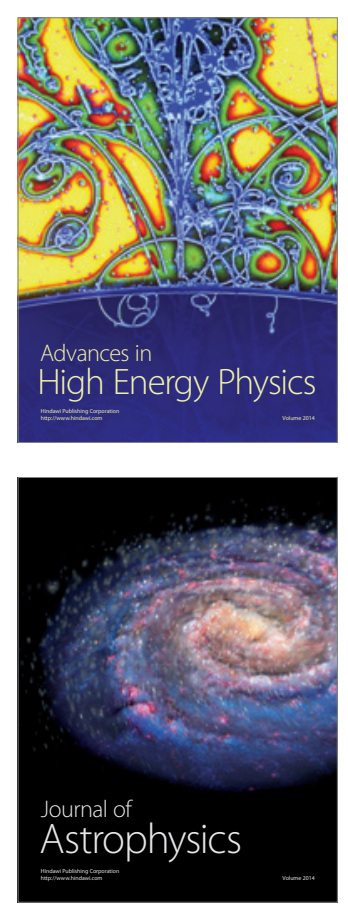
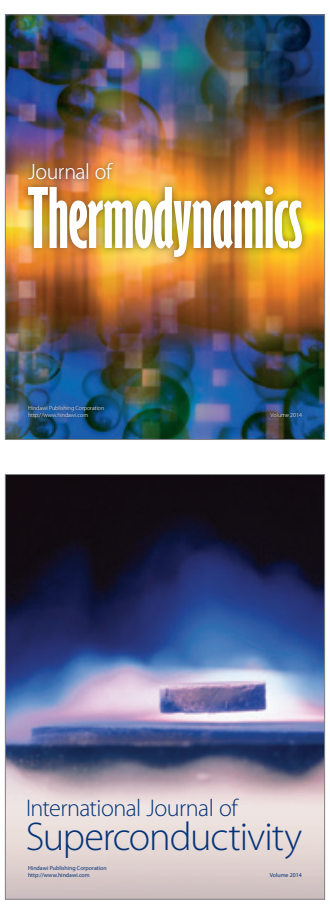
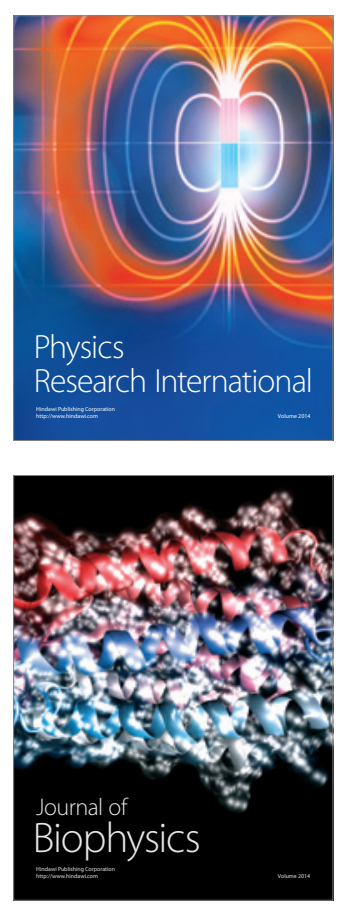
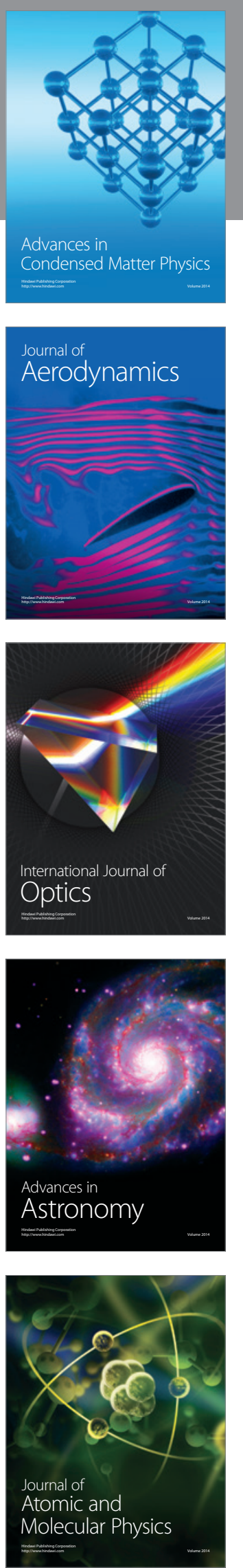